\title{
Medicina del adolescente
}

\author{
I. VILLAMIL CAJOTO, M. J. VILLACIÁN VICEDO'1, C. CANEDO ROMERO², \\ G. VILLACIÁN VICEDO ${ }^{3}$, M. RODRÍGUEZ FRAMIL
}

\author{
Servicio de Medicina Interna. ${ }^{I}$ Servicio de Pediatría. ${ }^{2}$ Unidad de Codificación. Hospital \\ Clínico Universitario. Santiago de Compostela. ${ }^{3}$ Estadístico. Universidad Complutense. \\ Madrid
}

\author{
MEDICINE IN THE ADOLESCENT
}

\section{RESUMEN}

Los numerosos cambios biológicos, cognitivos y socioculturales que suceden durante la adolescencia, podrían ser seguidos por Medicina Interna, pero el interés en las edades avanzadas es creciente y los más jóvenes están siendo tratados por otros especialistas. Presentamos los datos disponibles sobre adolescentes ingresados en nuestro Hospital y discutimos el interés de la Medicina Interna por los adolescentes.

Material y métodos: Revisión de los datos de los pacientes entre $15 \mathrm{y}$ 21 años ingresados durante el período 1996-2002.

Resultados: Ingresaron 5.894 pacientes adolescentes en este período, $1326(23 \%)$ en el Servicio de Medicina Interna de ellos 755 (57\%), eran varones. Las principales causas de ingreso fueron enfermedades infecciosas (fundamentalmente infecciones urinarias y de vías respiratorias). 160 pacientes $(6 \%)$ eran consumidores de tabaco.

Discusión: La adolescencia marca la transición a al edad adulta. La mayoría de los problemas de salud entre adolescentes resulta de accidentes (fundamentalmente de tráfico) pero existe un número importante de patologías que son sin duda tratadas por internistas en otros grupos de edad. Un número importante de pacientes es seguido por Pediatría y la "especialidad" de Medicina del Adolescente está constituida sobre todo por pediatras. Consideramos, como muestra este trabajo, que este grupo de edad podría ser seguido habitualmente por Medicina Interna pero parece necesario un mayor énfasis en la formación en estas edades. La oportunidad para incorporar adolescentes en la práctica de Medicina Interna es un desafío a tener en cuenta.

PALABRAS CLAVE: Adolescente. Medicina Interna.

\section{ABSTRACT}

The biologic, cognitive and sociocultural changes that occur during adolescence include a wide range such as to be best treated by internists, but focus in old ages is increasing and younger are being treated by others specialists. We review the data of those adolescents attended in our Hospital and discuss Internal Medicine interest in adolescents.

Material and methods: Epidemiological data, and clinical status of those patients between 15 and 21 years old admitted in our hospital during the period 1996-2002 were analysed. Results. 5894 admissions of adolescent's patients were made in this period. $1326(23 \%)$ where attended in the Internal Medicine Department. 755 (57\%) were male. Main causes of admission were infectious diseases, (mostly urinary tract and respiratory infections). Tobacco and alcohol abuse were present in 160 (6\%) patients.

Discussion: Adolescence marks the transition from childhood to adulthood. Health problems and deaths among adolescents and young adults result from accidents (mostly from motor vehicle), but there are conditions usually matter of our speciality. A significant number of patients are been attended by Paediatrics Department and such a speciality as Adolescence Medicine is mostly integrated by paediatric specialists. We consider as this paper show, these ages' ranges are clearly matter of our specialty and best treated by internists, but probably need a better consideration in our training. The opportunity to incorporate more adolescents into practice if realized, could prove challenging for internists.

KEY WORDS: Adolescent. Internal Medicine.

Villamil Cajoto I, Villacián Vicedo MJ, Canedo Romero C, Villacián Vicedo G, Rodríguez Framil M. Medicina del adolescente. An Med Interna (Madrid) 2005; 22: 525-528.

\section{INTRODUCCIÓN}

La adolescencia corresponde a la edad en la que se producen los intensos cambios físicos y psicosociales que generalmente se inician y terminan dentro de la segunda década de la vida (1). La Organización Mundial de la Salud (OMS), considera adolescencia entre los 10 y los 19 años y juventud al perí- odo entre los 19 y los 25 años de edad. Precisa sin embargo, se trata de una "fase" más que un período fijo en la vida de un individuo, una fase en la que el individuo no es ya un niño pero todavía no es un adulto (2). En cuanto a la asistencia sanitaria en nuestro país, los Servicios Hospitalarios y de Atención Primaria de Pediatría prestan asistencia médica a las personas de hasta catorce años de edad $(3,4)$. De acuerdo con

Trabajo aceptado: 20 de octubre de 2005

Correspondencia: Iago Villamil Cajoto. Servicio de Medicina Interna. Hospital Clínico Universitario. Choupana, s/n. 15706 Santiago de Compostela. A Coruña. e-mail: iago.villamil.cajoto@sergas.es 
el término empleado en el Index Medicus (Medical Subject Headings) la adolescencia corresponde al período de edad entre los 13 y los 18 años. La Society for Adolescent Medicine (SAM) de EE.UU. se encarga desde los años 60 del estudio de las personas de entre 10 y 25 años (5).

Aún con las dificultades en la consideración de la edad, y la tendencia al descenso en la natalidad en el mundo occidental, se trata de un grupo de pacientes importante en número y en demanda sanitaria. Según datos del Padrón de 2003 en España hay 41200600 habitantes, teniendo una distribución porcentual por edad en la franja de 16 a 39 años del 37,4\% con un índice de juventud (porcentaje de la población menor de 20 años con relación a la de 60 años o más) de 94,78 en 2002 (frente al 113,20 en 1996) (6). De ellos en nuestra Comunidad Autónoma con 2.695.880 habitantes, el grupo de 14 a 21 años de edad es de 128.219 individuos (4,7\% de la población de la Comunidad), según datos del Censo de 2001 (7). En cuanto a la morbilidad hospitalaria en España, en la población de 15 a 24 años los enfermos dados de alta por cada 1000 habitantes, fueron de 52,6 en 2002 frente a 57 de 1996 (en 2002 en el grupo de edades de 55 a 65 años la morbilidad hospitalaria fue de 126,5/1.000 habitantes y en el de 75 y más años de edad, de 281,7/1.000 habitantes) (8). En EE.UU. se produjeron aproximadamente 61,8 millones de visitas médicas ambulatorias en 1994 por parte de adolescentes entre 11 y 21 años (9). De acuerdo con el National Ambulatory Medical Care Survey de EEUU, solamente el 5,3\% de éstas de entre 12-17 años y el 7,4\% de los de entre 18-21 años se hicieron a internistas (10).Se estima que en EE.UU. hay unos cinco millones de ingresos anuales de adolescentes (11).

Parece existir poco interés por los adolescentes en Medicina Interna, estimándose que de lo publicado en revistas como Annals of Internal Medicine en 1998, menos del 5\% incluye como palabra clave adolescentes (9). Según nuestros datos no existen publicaciones sobre medicina del adolescente en Medicina Interna hasta el momento en nuestro país. Aquí la medicina del adolescente parece exclusiva de la Sociedad Española de Pediatría y sin embargo hay un gran número de pacientes vistos habitualmente en los Servicios de Medicina Interna. Presentamos los datos de ingresos en nuestro Centro comparando los Servicios de Medicina Interna y de Pediatría y analizamos la situación de la medicina del adolescente en Medicina Interna.

\section{PACIENTES, MATERIALES Y MÉTODOS}

Se realizó la revisión sistemática de los pacientes mayores de 14 años (final de la edad pediátrica en nuestro país) y pacientes hasta 25 años de edad, de acuerdo con los límites establecidos por la SAM, ingresados en el Complexo Hospitalario Universitario de Santiago de Compostela, en el período entre enero de 1996 y diciembre de 2001 (ambos incluidos). Se seleccionaron inicialmente todos los pacientes en estos grupos de edad, pero al comprobar que ningún paciente ingresado en pediatría era mayor de 21 años y de acuerdo con algunas definiciones indicadas previamente, se decidió comparar los grupos de edad entre 15 y 21 años (ambos incluidos) ingresados en estos servicios. Dadas las peculiaridades del Área de Pediatría, se incluyen los servicios de los que consta: Pediatría con sus distintas Unidades, Cirugía Pediátrica y UCI Pediátrica. Se evaluaron las características epidemiológicas y los motivos de ingreso de los pacientes comparándose ambos Servicios.

\section{RESULTADOS}

Durante el periodo de estudio ingresaron en nuestro centro 9789 pacientes de edades comprendidas entre 15 y 25 años (2.428, el 25\%, en Medicina Interna). El 75\% de éstos, eran menores de 23 años, la media de edad es de 20 años con una mediana igualmente de 20 años y la moda 23 años. En cuanto a la distribución por sexo, $4.970(50,8 \%)$ eran varones.

De estos pacientes entre 15 y 25 años, 5.894 (61\%) eran menores de 21 años. En cuanto a los menores de 21 años objeto de este estudio, los datos pueden verse en las tablas I y II. Los pacientes ingresados en el área de pediatría fueron 1058, (18\% de los menores de 21 años). El 99\% (1.050 pacientes) eran menores de 19 años. Ingresaron 626 mujeres (59,2\%). Los ingresos en UCI Pediátrica corresponden a 102 pacientes (9,6\% de los ingresos en pediatría). Entre las causas más frecuentes de ingreso destacan las incluidas en la tabla I.

\section{TABLA I}

ADOLESCENTES INGRESADOS EN PEDIATRÍA

\begin{tabular}{|c|c|c|c|}
\hline Sexo & Edad & $\begin{array}{l}\text { Diagnósticos } \\
\text { más frecuentes }\end{array}$ & \\
\hline $\begin{array}{l}\text { Mujer: } 626(59 \%) \\
\text { Hombre: } 342(41 \%)\end{array}$ & $\begin{array}{l}\text { Media: } 15,7( \pm 1) \\
\text { Mediana: } 15 \\
1.050 \text { pacientes }(99 \%), \\
\text { eran menores de } \\
19 \text { años }\end{array}$ & $\begin{array}{l}\text { Neoplasia: } \\
\text { Anemia:43 (4\%) } \\
\text { Anorexia nerviosa: } \\
\text { Infección: } \\
\text { Fracturas: }\end{array}$ & $\begin{array}{l}85(8 \%) \\
38(3,5 \%) \\
37(3,5 \%) \\
18(2 \%)\end{array}$ \\
\hline
\end{tabular}

Neoplasia: incluye órgano sólido y hematológicas.

Anemia: incluye anemias ferropénicas y posthemorrágicas.

Infección: incluye: neumonía, bronquitis, Infección urinaria, mononucleosis infecciosa

En cuanto a Medicina Interna, 1.327 pacientes de edades entre 15 y 21 años ingresaron en este periodo, el 23\% de los pacientes de estas edades. La media de edad fue de 18,3 años $( \pm 1,3$ años).En cuanto a la distribución por sexos, 755 pacientes $(57 \%)$ eran mujeres (Tabla II). Las causas de ingreso fue-

\section{TABLA \|}

\begin{tabular}{llll}
\multicolumn{3}{c}{ ADOLESCENTES INGRESADOS EN MEDICINA INTERNA } \\
\hline Sexo & Edad & $\begin{array}{l}\text { Diagnósticos } \\
\text { más frecuentes }\end{array}$ \\
\hline Mujer: $755(57 \%)$ & Media: $18,3( \pm 1,3)$ & Procesos infecciosos: & $85(8 \%)$ \\
Hombre: $570(43 \%)$ & Mediana: 18 & Reagudización asma: & $43(4 \%)$ \\
& & Infección urinaria: & $38(3,5 \%)$ \\
& El $80 \%$ eran menores & Intoxicación: & $37(3,5 \%)$ \\
& de 20 años & Intento autolitico: & $18(2 \%)$ \\
& & Tuberculosis: & $25(1,8 \%)$
\end{tabular}

Procesos infecciosos: incluye bronquitis aguda, mononucleosis, meningitis, Gastroenteritis, Fiebre de origen vírico.

Intoxicación incluye: medicamentos, opiaceos, cocaína.

Tuberculosis: incluye pulmonar y extrapulmonar 
TABLA III

COMPARATIVA MEDICINA INTERNA-PEDIATRÍA

\begin{tabular}{lcc}
\hline & $\begin{array}{l}\text { Medicina Interna } \\
\left(n^{\circ} \text { pacientes }\right)\end{array}$ & $\begin{array}{l}\text { Pediatría } \\
\left(n^{\circ} \text { pacientes }\right)\end{array}$ \\
\hline Grupos de edad & & \\
15 años & 93 & 576 \\
16 años & 204 & 284 \\
17 años & 168 & 146 \\
18 años & 327 & 22 \\
19 años & 173 & 22 \\
20 años & 194 & 7 \\
21 años & 256 & 1 \\
\hline Total & 1.326 & 1.058 \\
\hline
\end{tabular}

ron fundamentalmente las detalladas en la tabla II. Se comparan los grupos de edad en Medicina Interna y Pediatría en la tabla III. En ambos grupos la suma de pacientes con hábito tabáquico era de 160 pacientes $(6 \%)$.

Otros servicios del área médica en los que ingresaron pacientes entre 15 y 21 años de edad, se detallan en la tabla IV. Además, 306 pacientes ingresaron en la Unidad de Desórdenes Alimentarios (se trata de la única unidad de este tipo en la Comunidad Autónoma por lo que los ingresos son de toda la autonomía).

\section{TABLA IV}

INGRESOS EN OTROS SERVICIOS ÁREA MÉDICA

\begin{tabular}{lc}
\hline Servicio & $N^{\circ}$ de pacientes \\
\hline Cardiología & 127 \\
Dermatología & 139 \\
Digestivo & 235 \\
Neumología & 606 \\
Neurología & 509 \\
Reumatología & 131 \\
Endocrinología & 136 \\
Hematología y Oncología médica & 518 \\
\hline Total & 2.919 \\
\hline
\end{tabular}

\section{DISCUSIÓN}

La adolescencia marca la transición entre la infancia y la edad adulta. La magnitud en las variaciones en lo físico, cognitivo, psicológico y social condicionan las dificultades de abordaje en la relación médico paciente con este colectivo. En la situación de enfermedad, conseguir empatía con el paciente es si cabe más complejo. El internista es un especialista que aporta una atención global al enfermo, con una visión de sus problemas como un todo. Ésta le da un papel particularmente idóneo en aquellos casos de pacientes con pluripatología, situaciones cada vez más frecuentes debido al envejecimiento progresivo de la población general. Pero este perfil es además perfectamente adecuado para el seguimiento de los adolescentes.
Nuestro estudio y lo mencionado anteriormente, pone de manifiesto que un número significativo de adolescentes está siendo atendido por pediatras pero muchos de éstos están siendo vistos habitualmente por las especialidades del área médica, y especialmente por los Servicios de Medicina Interna como se demuestra en los resultados. Sin embargo la formación específica en medicina del adolescente está siendo mayoritariamente llevada por pediatras. Desde el punto de vista de la Medicina Interna parece existir cierta pasividad e indiferencia. No existen grupos de trabajo de la Sociedad Española de Medicina Interna en adolescentes y por ejemplo en los congresos nacionales e internacionales de la especialidad como en el reciente vigésimo séptimo Congreso Mundial de Granada, no existían categorías de abstracts en adolescentes para las comunicaciones al congreso; sí existían sin embargo categorías sobre gerontología y geriatría. Esta comunicación preliminar fue aceptada en la categoría Preventive Medicine \& Public Health en éste Congreso. Este abandono de lo que creemos una de las áreas más propias de Medicina Interna, hace necesaria una reflexión profunda sobre esta situación. La Medicina Interna es por sus características de formación, la especialidad que más y mejor puede y debe tratar a los pacientes de estos grupos de edad. Pero es imprescindible en nuestra opinión, una puesta al día importante en la formación específica en estos pacientes.

La OMS establece como prioridades en su política de estrategia global de salud, la promoción de la salud en niños y adolescentes (2).Una característica general de la población adolescente, es la vulnerabilidad y el cuestionamiento de la autoridad de las figuras de referencia hasta la infancia. En esta etapa, al tiempo que se exige mayor independencia, la adquisición de responsabilidad sobre su salud es gradual, de forma que en gran medida el acceso a la información y a los servicios sanitarios está limitado. Esta fase hace que la eliminación en las referencias en pautas de comportamiento como vía de autoafirmación, haga surgir la adquisición conductas de riesgo (dietéticas, hábitos tóxicos, sexuales, accidentes, etc.). Por ello la OMS establece como una de las grandes prioridades en el manejo de estos pacientes, que los Servicios que se encargan de su seguimiento, aumenten el papel en la información, apoyo psicosocial, promoción y prevención de la salud y no exclusivamente en la tarea de "curación" (2). La amplia variedad de procesos ingresados en nuestro estudio (los grupos más frecuentes no superan los 300 casos en total) hace que las diversas patologías ingresadas encajen perfectamente con el perfil de una especialidad multidisciplinar como Medicina Interna.

Las Unidades hospitalarias para la atención al adolescente son una realidad en EE.UU. desde los años 60 del pasado siglo. Para el establecimiento de éstas, varias sociedades científicas de EE.UU. (incluida la de Medicina Interna), establecieron unas guías aún en vigor (10). Se estima que deben existir unas 40-60 unidades de adolescente en EE.UU. y algunas más en Europa, Asia, América del Sur y Australia. El tamaño de las unidades es de entre 6 y 35 camas, aunque la mayoría tienen entre 11 y 20 camas (13), y los límites de edad entre los 10-13 años y los 17-24 años. Los aparentes inconvenientes económicos en la existencia de estas unidades, dada la tendencia generalizada a la reducción del gasto sanitario en todos los sistemas de salud, parecen estar fuera de duda (13), además de las notables ventajas en la situación de estos pacientes para una edad que generalmente se califica como "difícil" en la que 
la hospitalización con pacientes pediátricos o bien con ancianos podría resultar inconveniente.

La Sociedad Americana para la Medicina del Adolescente (SAM) tiene una gran actividad en EE.UU., figurando entre sus objetivos el cuidado de la salud, formación profesional, investigación en salud y cuidados en personas entre 10 y los 25 años, aplicándose estas materias tanto a la atención primaria como a la especializada. (5). Esta Sociedad es multidisciplinar y no está adscrita a ninguna otra, pero reconoce en sus temas de interés la incorporación de internistas en esta disciplina (14). La propia especialidad de Medicina Interna en EE.UU. reconoce su interés, y la Sociedad Americana de Medicina Interna incluye entre sus disciplinas para la formación de residentes la instrucción en adolescencia (15). Además entre sus planes de formación especializada (estudio

\section{Bibliografía}

1. Neinstein LS. Consolidation of psychosocial scales. J Adolesc Health Care 1988; 9: 507-11.

2. Southall DP, Burr S, Smith RD, Bull DN, Radford A, Williams A, Nicholson S. The Child-Friendly Healthcare Initiative (CFHI): Healthcare provision in accordance with the UN Convention on the Rights of the Child. Child Advocacy International. Department of Child and Adolescent Health and Development of the World Health Organization (WHO). Royal College of Nursing (UK). Royal College of Paediatrics and Child Health (UK). United Nations Children's Fund (UNICEF). Pediatrics 2000; 106: 1054-64.

3. Decreto ley 1360/66 de 23 de diciembre, artículo 21. Estatuto jurídico del personal facultativo ( $\mathrm{BOE} \mathrm{n}^{\circ} 132$ de 30 de diciembre de 1966).

4. Resolución de 23 de julio de 1998 del Ministerio de Sanidad y Consumo. Creación del pediatra de Atención Primaria. (BOE n 187 de 6 agosto de 1998).

5. A Position Statement of the Society for Adolescent Medicine. J Adolesc Health 1995; 16: 413.

6. Indicadores Sociales. Edición 2004 (en línea) (último acceso 15 agosto 2004). URL disponible en http://www.ine.es/daco/daco42/sociales.htm

7. Censo 2001. Población segundo sexo e idade (ano a ano) en Galicia. (en linea) (última consulta el 15 de agosto de 2004). URL disponible en: http://www.ige.xunta.es/ga/demograficas/censos/poboacion/index2001.htm. postgrado o fellowship) incluye la Medicina del Adolescente (16).

En nuestro país desde 1987 existe una Sociedad Española de Medicina del Adolescente de la Asociación Española de Pediatría que realiza actividades periódicas. La Sociedad Española de Medicina Familiar y Comunitaria cuenta desde 1998 con un Grupo de trabajo de adolescencia. Pero la Medicina Interna española parece ajena a este grupo de edad que en el futuro puede ser especialmente importante dado el nuevo pico de natalidad evidenciado en la última encuesta nacional de población (6). Pero además es sobre el que más se puede incidir para tratar ya y prevenir muchas de las patologías sobre las que se está demostrando excelente formación en el paciente adulto. Tal vez es momento de plantearse esta tarea.

8. Indicadores Sociales. Edición 2004 (en línea) (última consulta 15 agosto 2004). URL disponible en: http://www.ine.es/daco/daco42/sociales.htm

9. Joffe A. Why Adolescent Medicine? Med Clin North Am. 2000; 84: 769-85.

10. Ziv A, Boulet JR, Slip GB: Utilization of physician offices by adolescents in the United States. Pediatrics 1999; 104: 35-42.

11. McManus M, McCarthy E, Kozac LJ, Newacheck P. Hospital use by adolescents and young adults. J Adolesc Health Care 1991; 12: 10715.

12. Committee on inpatient care for adolescents of the Society for adolescent Medicine. Characteristics of an inpatient unit. Clin Pediatr 1973; 12: 17-21.

13. Fisher M. Adolescent in patient units. Arch Dis Child 1994; 70: 461-3.

14. Graves CE, Bridge MD, Nyhuis AW. Residents' perception of their skill levels in the clinical management of adolescent health problems $\mathbf{J}$ Adolesc Health Care 1987; 8: 413-8.

15. Djuricich AM. Teaching medical residents about teenagers: an introductory curriculum in adolescent medicine. Acad Med 2002; 77 : 745-6.

16. Health care needs of the adolescent. American College of Physicians. Ann Intern Med 1989; 110: 930-5. 